\title{
Effect of repetitive ultraviolet irradiation on the physico-chemical properties and microbial stability of pineapple juice
}

\begin{abstract}
The study aims to investigate the effect of repetitive ultraviolet irradiation (UVÏ UV) and the combination effect with dimethyl dicarbonate (UVï DMDCï UV) on the physico-chemical properties and microbiological stability of pineapple juice. UV dosages of $10.76 \mathrm{~mJ} / \mathrm{cm} 2$ percycle and $250 \mathrm{ppm}$ of DMDC were used. There was a significant decrease in turbidity, total phenolic and vitamin $\mathrm{C}$ in the treated juices. The UVÏUV reported a significant reduction of $1.91 \log \mathrm{CFU} / \mathrm{ml}$ in total plate count and $1.4 \log \mathrm{CFU} / \mathrm{ml}$ in yeast and mould. Post addition of DMDC into the UV irradiated juice (UVï UVï DMDC) showed reductions of $2.61 \log$ CFU/ml for TPC and $4.87 \log$ CFU/ml for YM. This study demonstrated the effectiveness of UV irradiation in preserving the nutritional quality and the addition of DMDC can have a combination effect with the UV irradiation of juice in terms of microbial reduction. However, the treatments were not sufficient to achieve adequate microbial reduction as required by the FDA.

Industrial relevance: Dimethyl dicarbonate (DMDC) is one of the effective anti-microbial agents that can control a wide range of microorganisms which includes Escherichia coli 0157:H7 and yeast. The effect of dimethyl dicarbonate (DMDC) in reducing microbial counts was significant in this study. According to Threlfall and Morris (2002), DMDC is used to prevent fermentation in excessive yeast contamination in wine production. Moreover, Halim et al. (2012) stated that DMDC has shown promising results for microbial inactivation of fruit juices in a preliminary study in lab. Therefore, combination effect with additives (DMDC) may be able to increase the efficiency of the UV irradiation for microbial reduction in juice and longer the shelf life of juice.
\end{abstract}

Keyword: Ultraviolet irradiation; Juice; Dimethyl dicarbonate 